\title{
HELMET MOUNTED UHF ANTENNA FOR MOBILE USER OBJECTIVE SYSTEM (MUOS)
}

\author{
Richard W. Adler \\ David S. Gibbs \\ Jovan E. Lebaric \\ Stephen S. Schwarz \\ Naval Postgraduate School \\ Department of Electrical Engineering \\ Monterey, CA
}

\begin{abstract}
The Mobile User Objective System (MUOS) is the Navy's next generation narrowband tactical communications system that will provide a significant increase in capacity and link availability to disadvantaged users, including handheld terminals. Future MUOS antennas will have a receive band of $243 \mathrm{MHz}-270 \mathrm{MHz}$ and a transmit band of $292 \mathrm{MHz}-317 \mathrm{MHz}$ with a voltage standing wave ratio (VSWR) of less than three across both bands. Additionally, the antenna should be circularly polarized with a nearly omni-directional radiation pattern above 10 degrees in elevation for optimum satellite coverage. A helmet-mounted antenna that can meet the space and weight constraints imposed by the application is a twoturn spiral with a different pitch to each turn. Our design is a two-arm Archimedes spiral encapsulated in a nylon shell that fits over the standard-issue US Kevlar helmet. The antenna was modeled and simulated using Ansoft's High Frequency Structure Simulator (HFSS) software. A half-power beamwidth of 120 degrees, a front-to-back (zenith-to-nadir) ratio of $3.6 \mathrm{~dB}$, and a VSWR of less than three were achieved using a feed port impedance of 150 ohms. Recent work includes specific absorption rate (SAR) calculations and the addition of a reflector ring, which increases the front-to-back ratio and reduces the feed port impedance to the standard 50 ohms.
\end{abstract}

\section{INTRODUCTION}

Ultra High Frequency (UHF) communications are highly desirable for military use, especially the mobile warfighter, due to its ability to penetrate heavy weather, foliage, and concrete buildings. US Navy narrowband tactical satellite communications are currently handled by the UHF Follow-On (UFO) constellation, first launched in 1993. The Mobile User Objective System (MUOS) has been designated to supplement, and eventually replace, the current UFO constellation. The primary objectives of MUOS are to provide assured access to communications worldwide, point-to-point and netted communications, joint interoperability, and communications on the move [1].

Future MUOS ground user terminal antennas should be highly mobile and maintain a low profile. This paper presents a semi-conical two-arm Archimedes spiral antenna encapsulated in a nylon shell that fits directly over the standard-issue US ground troop Kevlar helmet. This design is well suited for the required application due to its low profile and the protection provided to the antenna by the nylon encapsulation. Additionally, the antenna and its nylon shell can be easily retrofitted to another helmet. The geometry of the spiral antenna is presented along with the impact on performance due to the nylon encapsulation. Results of computer simulations are used to analyze antenna performance in the key areas of Voltage Standing Wave Ratio (VSWR), radiation pattern, and zenith-to-nadir ratio of the radiation pattern. Finally, on-going work is presented.

\section{HF HELMET ANTENNA DESIGN}

The basic design of the helmet antenna is shown in Figure 1 and Figure 2. The spiral antenna is embedded within a $1.5 \mathrm{~cm}$ thick nylon shell (not shown) that fits over the helmet. The helmet was created using Rhinoceros

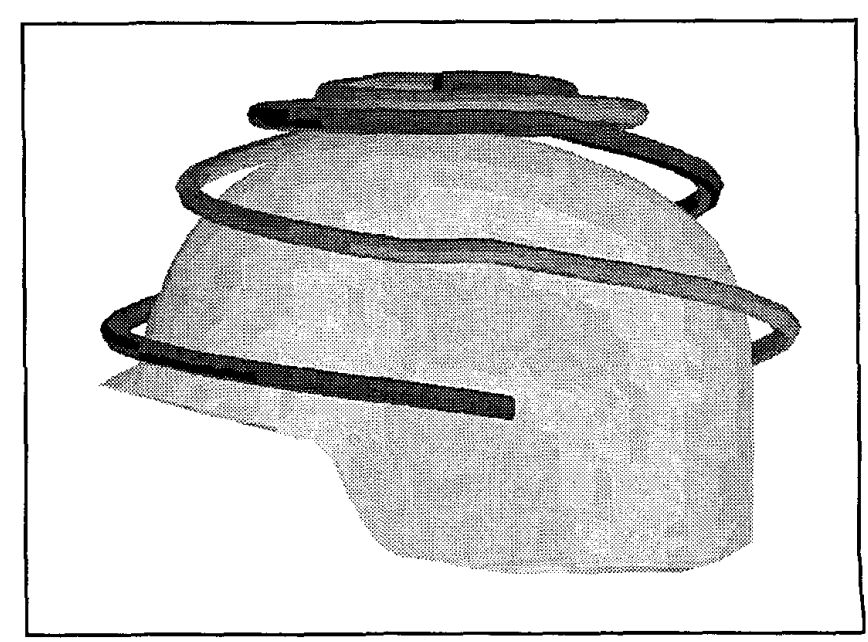

Figure 1. Spiral antenna and helmet (side view). 


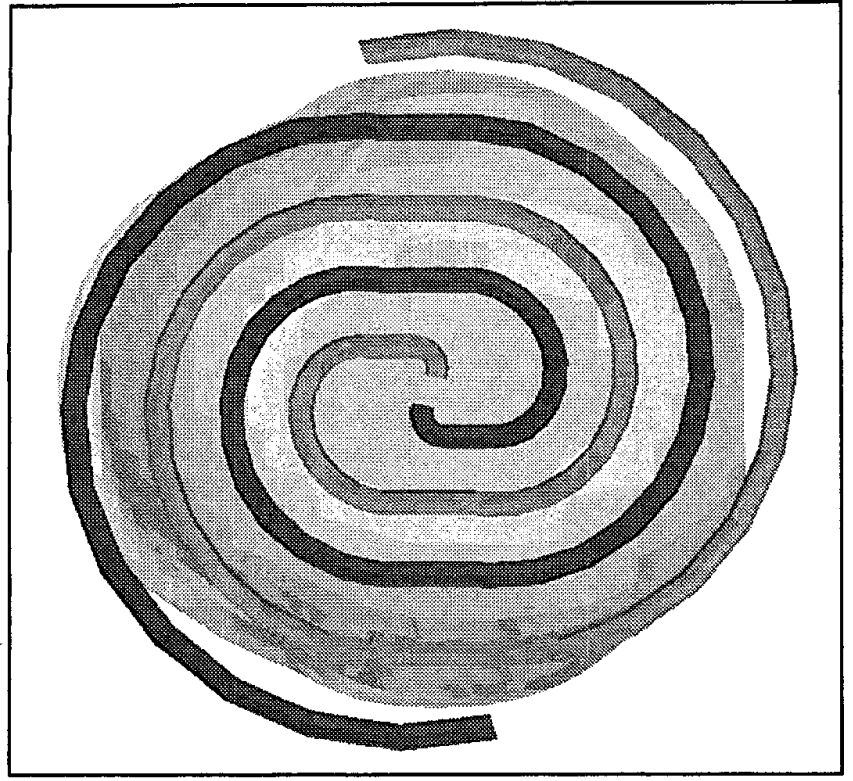

Figure 2. Spiral antenna and helmet (top view).

software and a MicroScribe digitizer stylus on an actual US ground troop helmet. The model was then imported into High Frequency Structure Simulator (HFSS) and swept inward ("extruded") to create a helmet of $1 \mathrm{~cm}$ thickness.

A spiral antenna was chosen over a dipole or patch antenna because its shape readily conforms to the helmet, it does not require a ground plane, and it is the closest to being an effective radiator because it naturally has the longest length. Of several designs simulated with a varying number of turns and a varying wire diameter, the best solution was a semi-conical asymmetric antenna composed of two "stacked" Archimedes spirals, with the turns at the top of the helmet of one fixed pitch and the turns at the bottom of a different fixed pitch [2]. The pitch is defined as the distance between successive turns of the spiral. Due to the asymmetry of the helmet from front to rear and its elliptical shape, the spirals were designed using spiral segments joined together by $3.5 \mathrm{~cm}$ long straight segments. The addition of the straight segments actually extends the completed spiral by another quarter turn. The two arms of each of the two "stacked" spirals are identical, except for the 180-degree rotation. Each spiral is composed of a $1 \mathrm{~cm}$ diameter coaxial cable. The feed port is located at the top of the antenna; the center conductor of one spiral is soldered to the other spiral's outer conductor at the top of the antenna.

The nylon shell serves several purposes. Nylon is lightweight, resistant to abrasion and wear, and has excellent tensile strength. The nylon holds the spiral in the proper shape and protects against damage. Additionally, nylon has a dielectric constant of 4.3 , which has the effect of slowing the speed of the traveling waves and making the antenna appear electrically longer.

\section{SIMULATION RESULTS}

The three primary parameters analyzed were the VSWR across the operating bandwidth of the antenna, the radiation pattern, and the zenith-to-nadir power ratio, which will hereafter be referred to as the front-to-back ratio.

A VSWR of less than three is desirable because this means that no more than 25 percent of the power is reflected back to the source. The minimum frequency of interest is $243 \mathrm{MHz}$, corresponding to a $1.23 \mathrm{~m}$ wavelength. The longer the antenna is with respect to the wavelength the better it performs at that wavelength, but creating a long antenna within a small area around the helmet is difficult. Reducing the pitch of the turns in the spiral antenna allows more turns and creates a longer antenna which would normally improve VSWR performance at lower frequencies, but the smaller spacing between the arms of the spiral creates a larger electromagnetic coupling; the increased coupling leads to a larger VSWR "ripple", with the VSWR exceeding the limit at certain frequencies within the operating band [2]. An alternative method for increasing the antenna electrical length has been employed; the nylon shell is used to clad the antenna and slow down the electromagnetic wave propagation along the spiral, therefore effectively increasing the antenna electrical length.

Figure 3 shows the impact on VSWR performance of using the nylon shell as opposed to the same antenna

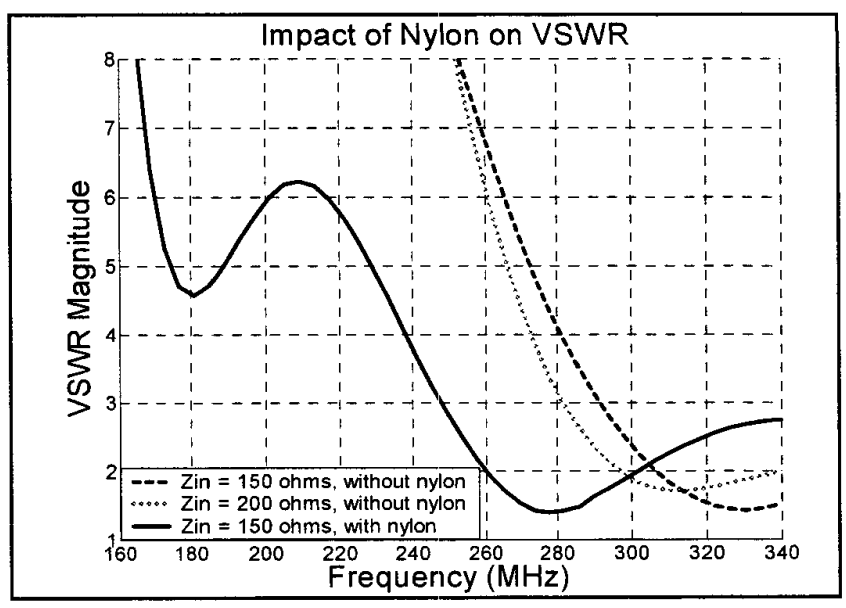

Figure 3. VSWR performance comparison. 
without the shell. The antenna embedded in nylon performed best with a reference impedance of $150 \mathrm{ohms}$; the VSWR is lower than 3 above $246 \mathrm{MHz}$. The dielectric loading of the nylon provided an additional 30 $\mathrm{MHz}$ of bandwidth at the low frequency end as compared to the same antenna without the nylon shell. For the antenna without the nylon shell, a reference impedance of 200 ohms gives better VSWR performance than a reference impedance of $150 \mathrm{ohms}$, but the improvement is very small compared to the impact of adding the nylon shell.

The radiation pattern at $300 \mathrm{MHz}$ is shown in Figure 4 . An angle of theta $=0$ degrees is directed straight upwards from the antenna, theta $=180$ degrees is toward the ground (operator), and theta $=90$ degrees is off to the right side of the operator. The half-power beamwidth is 120 degrees, providing fairly broad coverage above the helmet. The asymmetrical design of the spiral provides a front-to-back ratio of $3.6 \mathrm{~dB}$, meaning approximately $70 \%$ of the radiated power is radiated upwards and $30 \%$ of the power is radiated down toward the operator. This asymmetrical design provided a $1.8 \mathrm{~dB}$ increase in the front-to-back ratio over a perfectly symmetrical spiral design [2].

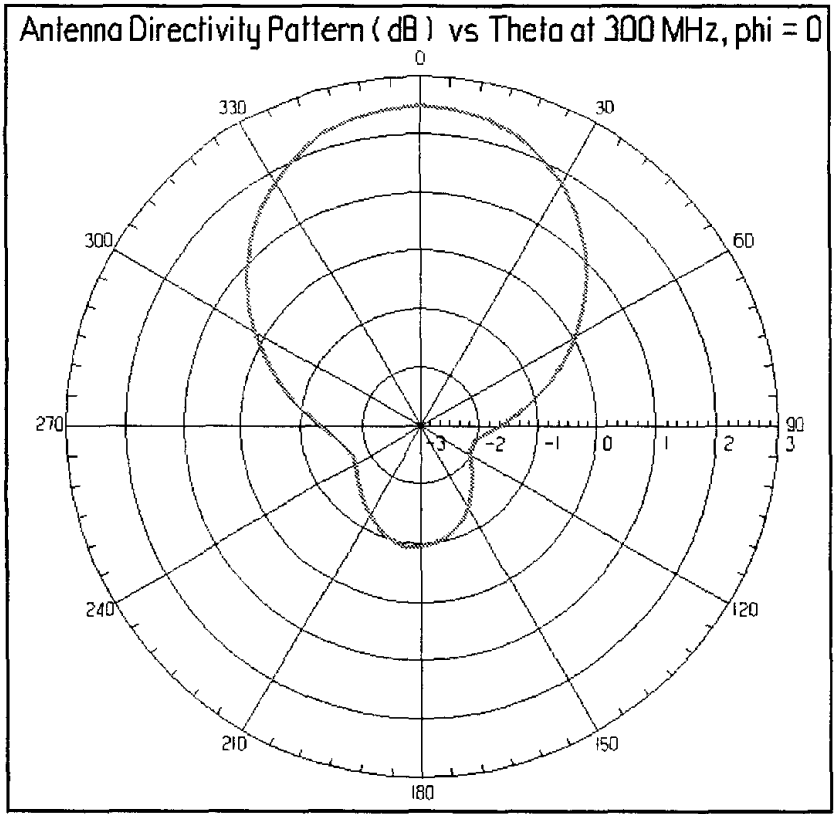

Figure 4. Radiation pattern for transmit band.

These results were obtained using HFSS Version 7.0.04 and later duplicated using HFSS Version 8.0.25 on a different computer. Previous research performed by Dr. Lebaric, Dr. Adler, and Mathew Limbert showed a strong correlation between impedance and VSWR measurements of a vest antenna compared to HFSS simulation results [3], so there is a fairly high level of confidence in the accuracy of the simulation results.

\section{CURRENT RESEARCH}

Current research is focused on increasing the front-toback ratio and determining the Specific Absorption Rate (SAR). Based upon the principles of a Yagi-Uda antenna, a reflector ring partially embedded within the nylon shell near its base improved the front-to-back ratio by an additional $6 \mathrm{~dB}$ in the HFSS model; however, this design is undesirable because the non-embedded portion of the reflector ring wraps around the front of the helmet and impacts the operator's field of vision.

CST Microwave Studio is being used for current research because it contains an interface to a human body model, "HUGO", with which the SAR can be determined. An antenna on helmet model, similar to the HFSS model without the reflector ring, was designed and simulated within Microwave Studio; the simulation results were comparable to those obtained in HFSS although the frontto-back ratio was only slightly over $2 \mathrm{~dB}$ in the Microwave Studio model as compared to $3.58 \mathrm{~dB}$ in the HFSS model. Adding a reflector ring, embedded entirely within the nylon shell, increased the front-to-back ratio to approximately $4 \mathrm{~dB}$. Additionally, the optimum input impedance was reduced from $150 \mathrm{ohms}$ to 50 ohms, eliminating the need for a step-up transformer. Front-toback ratio improvement is limited because the geometric confines of the helmet and spiral do not allow optimum positioning of the reflector. To provide more flexibility in design and positioning of the ring, the pitch of the spiral was reduced in order to compact the vertical size of the antenna and free up space near the bottom of the helmet. Although the tighter coupling of the spiral arms creates the VSWR "ripple", it is believed that the addition of the human body will compensate for any loss in VSWR performance due to the increased coupling.

Figure 5 and Figure 6 on the following page show a typical design that includes all the major elements, including a $2 \mathrm{~cm}$ thick nylon shell and the human body. Memory requirements limited the selection of the human body to above the shoulders only. A simpler helmet, generated through the drawing interface, replaces the digitized helmet in order to reduce memory requirements and decrease simulation time.

Simulations were done with and without the human body; Figure 7 shows a comparison of VSWR performance, both relative to a reference impedance of 50 ohms. Note that some VSWR performance was sacrificed in 


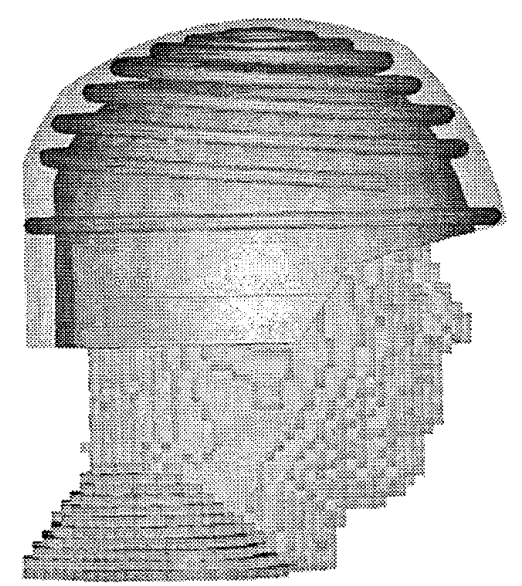

Figure 5. Full model (side view).

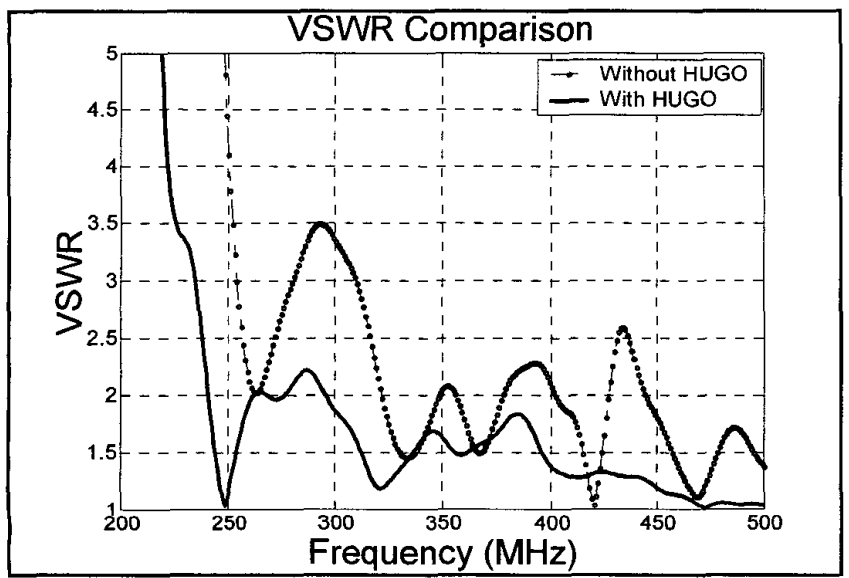

Figure 7. VSWR performance comparison.

compacting the size of the antenna; the result for the simulation without the body shows that the VSWR is below 3 at just above $250 \mathrm{MHz}$ and that there is a fairly wide local peak of magnitude 3.5 within the transmit frequency range. Insertion of the human body regains 25 $\mathrm{MHz}$ at the lower frequency range and reduces the VSWR peak to just over 2. The VSWR remains below 2.5 up to at least $1 \mathrm{GHz}$.

The radiation pattern for both simulations at $300 \mathrm{MHz}$ is shown in Figure 8. The orientation of the graph is: 0 degrees is straight up, 90 degrees is to the front or rear of the operator, and 180 degrees is toward the ground. The addition of the human body. in this model increased the front-to-back ratio by approximately $3 \mathrm{~dB}$, although the beamwidth has been reduced to approximately 90 degrees.

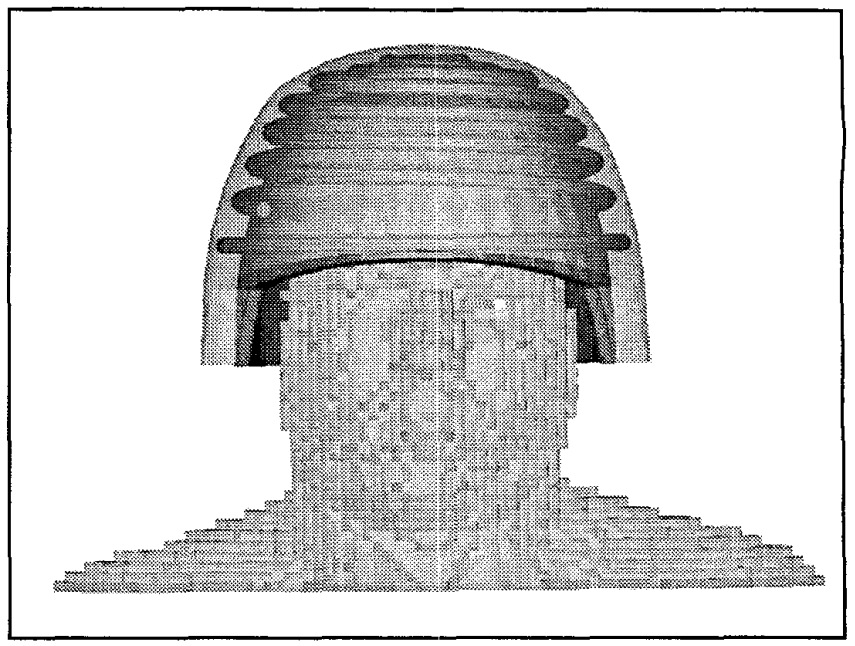

Figure 6. Full model (front view).

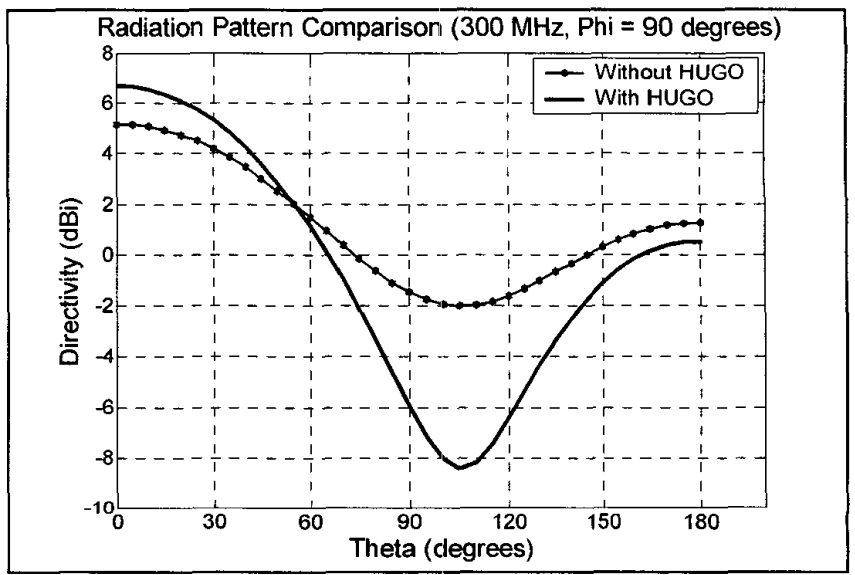

Figure 8. Radiation pattern.

Based on an average input power of 0.5 watts, the maximum SAR is $0.692 \mathrm{~W} / \mathrm{kg}$ as measured over any 1 gram of body tissue. The total average SAR is $0.04 \mathrm{~W} / \mathrm{kg}$ over the mass of the body in the model. Figures 9,10 , and 11 show representative cut-plane views of the SAR at $300 \mathrm{MHz}$; the red areas correspond to the highest SAR within that region of the body. The Federal Communications Commission (FCC) and Department of Defense (DoD) limits for radio-frequency exposure are $1.6 \mathrm{~W} / \mathrm{kg}$ as measured over any 1 gram of tissue and 0.08 $\mathrm{W} / \mathrm{kg}$ as averaged over the entire body. Rough calculations based on simulation results show that an EIRP of approximately $1 \mathrm{dBW}$ can be attained without exceeding the FCC and DoD limits for uncontrolled areas.

Further modifications to the antenna and reflector are being simulated, particularly the use of a two level (tiered) reflector ring to maximize the reflector 
circumference and its distance to the antenna, with an overall goal of maximizing the EIRP and minimizing the SAR. Material changes to the nylon shell are also being investigated as well as the incorporation of a resistive cloth lining on the inner surface of the helmet to reduce the SAR.

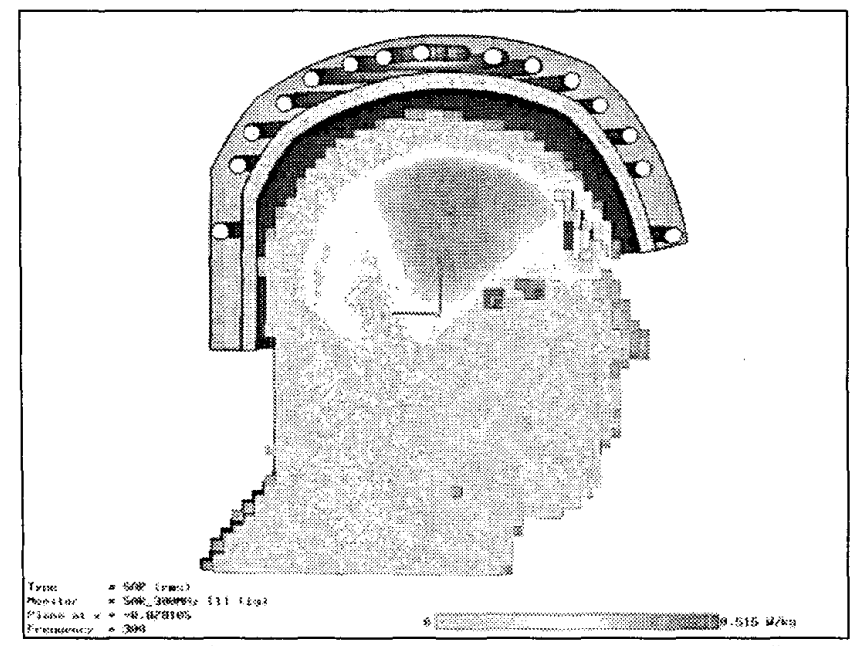

Figure 9. Side view of SAR (maximum $=0.515 \mathrm{~W} / \mathrm{kg}$ ).

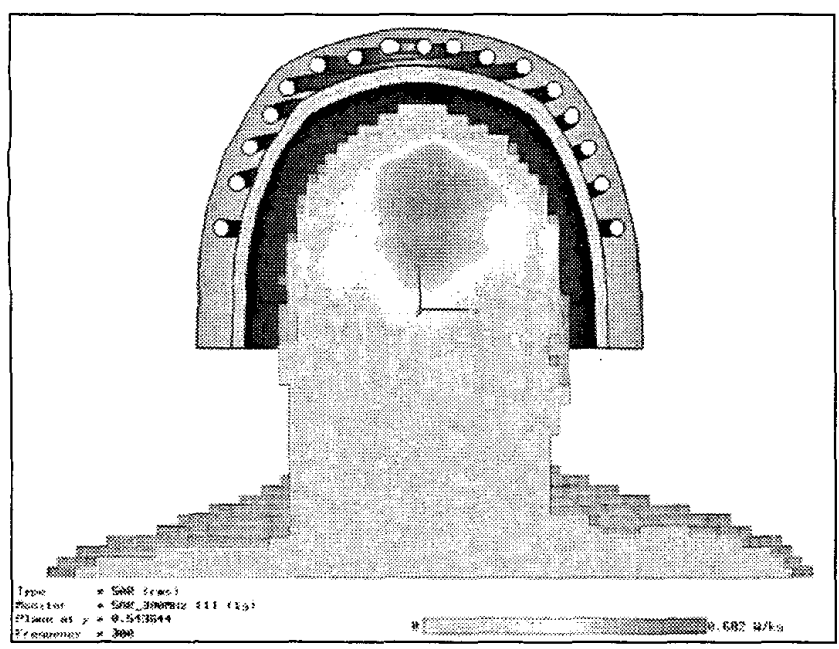

Figure 11. Front view of SAR (maximum $=0.682 \mathrm{~W} / \mathrm{kg}$ ).

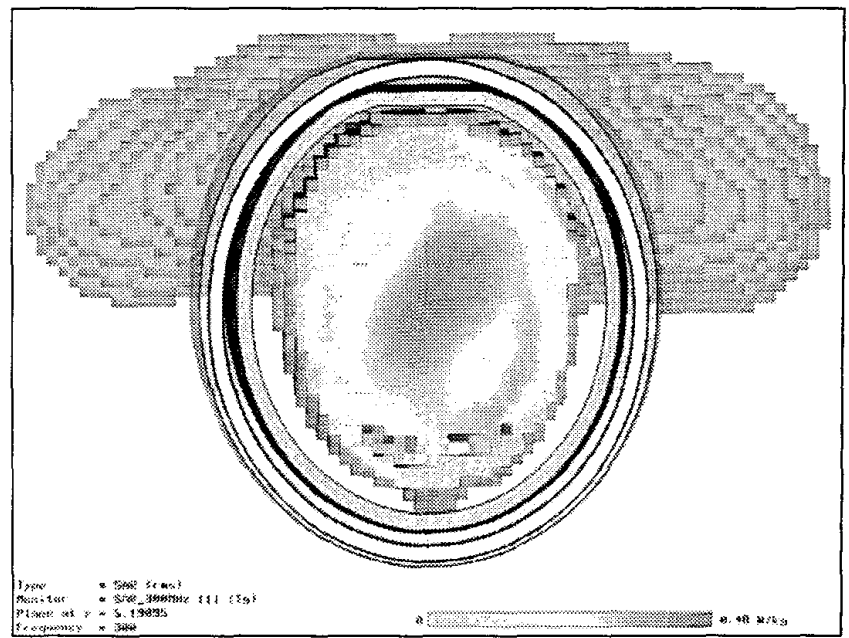

Figure 10. Top view of SAR (maximum $=0.48 \mathrm{~W} / \mathrm{kg}$ ).

\section{REFERENCES}

[1] Loiselle, J., Tarleton, R., and Ingerski, J., "The Next Generation Mobile User System (MUOS)." hitp//enterprise.spawar.navy.mil/spawarpublicsite/docs/ next_gen_muos.pdf.

[2] Gibbs, D.S., "Design of Mobile User Objective System (MUOS) Helmet Mounted UHF Antenna." Master's thesis, Naval Postgraduate School, 2001.

[3] Lebaric, J.E., Adler, R.W., and Limbert, M.E., "UltraWideband, Zero Visual Signature RF Vest Antenna for Man-portable Radios," Military Communications Conference, 2001, IEEE, Volume 2, pp. 1291-1294, 2001. 